\title{
A MERLIN movie of mass-loss from RT Vir
}

\author{
A. M. S. Richards, R. J. Cohen, I. Bains \\ N.R.A.L., Jodrell Bank, University of Manchester, U.K.
}

\section{J. A. Yates}

Department of Physical Sciences, University of Hertfordshire, U.K.

\begin{abstract}
We used MERLIN to observe RT Vir at $22 \mathrm{GHz}$ at six epochs during 10 weeks. The water maser emission comes from a thick expanding shell with an elliptical velocity field. MERLIN has a velocity resolution of $0.1 \mathrm{~km} \mathrm{~s}^{-1}$ and milli-arcsecond angular resolution, revealing details within the individual maser clouds, typically 12 mas in diameter spanning 15 velocity channels. The brightest peak doubles in intensity to 800 $\mathrm{Jy} /$ beam. Features at velocities close to the stellar velocity show the largest proper motions of $\sim 3$ mas away from the centre of emission. Some features are seen near the outer limits to the maser shell at early epochs only, but new masers appear close to the inner rim. The variability of individual maser features is not a simple function of the stellar luminosity.
\end{abstract}

\section{Observations of RT Vir}

RT Vir is a semi-regular variable star (SRb) (Kholopov et al. 1985) which may be in a pre- or post- Mira phase (Szymczak et al. 1998), or an AGB star from a distinct population (Whitelock et al. 1994). Optical data provided by the AAVSO and AFOEV ${ }^{1}$ shows irregular variations between mag. 9.3 - 8.2. Fig. 1a shows its state during our radio observations. It is classed as an unsolved variable by HIPPARCOS; the parallax gives a distance of $140 \pm 20 \mathrm{pc}$. The mass loss rate estimated from $\mathrm{CO}$ observations is $3 \times 10^{-6} \mathrm{M}_{\odot} \mathrm{yr}^{-1}$ (Bujarrabal et al. 1989). The stellar velocity $V_{*}$ is $18.2 \mathrm{~km} \mathrm{~s}^{-1}$ (Nyman et al. 1986) which is close to the midpoint of its strong $\mathrm{H}_{2} \mathrm{O}$ maser emission, weak $\mathrm{OH}$ mainline masers, and CO (e.g. Mendoza-Torres et al. 1997; Kirrane 1986; Stanek et al. 1995).

RT Vir was observed using MERLIN ${ }^{2}$ at $22 \mathrm{GHz}$ at epochs $1-6$ during April - June 1996. Details are given in Table 1 along with a 1994 MERLIN observation (Bains 1995). The maximum baseline is $217 \mathrm{~km}$ giving $\lambda / d \approx 12$ mas. A bandpass of $2 \mathrm{MHz}$ was divided into 256 channels providing a velocity

\footnotetext{
${ }^{1}$ This research has made use of the databases of these organisations of variable star observers, which we acknowledge with thanks.

${ }^{2}$ The Multi Element Radio Linked Interferometer Network, operated by the University of Manchester on behalf of PPARC http://www.jb.man.ac.uk/merlin/
} 

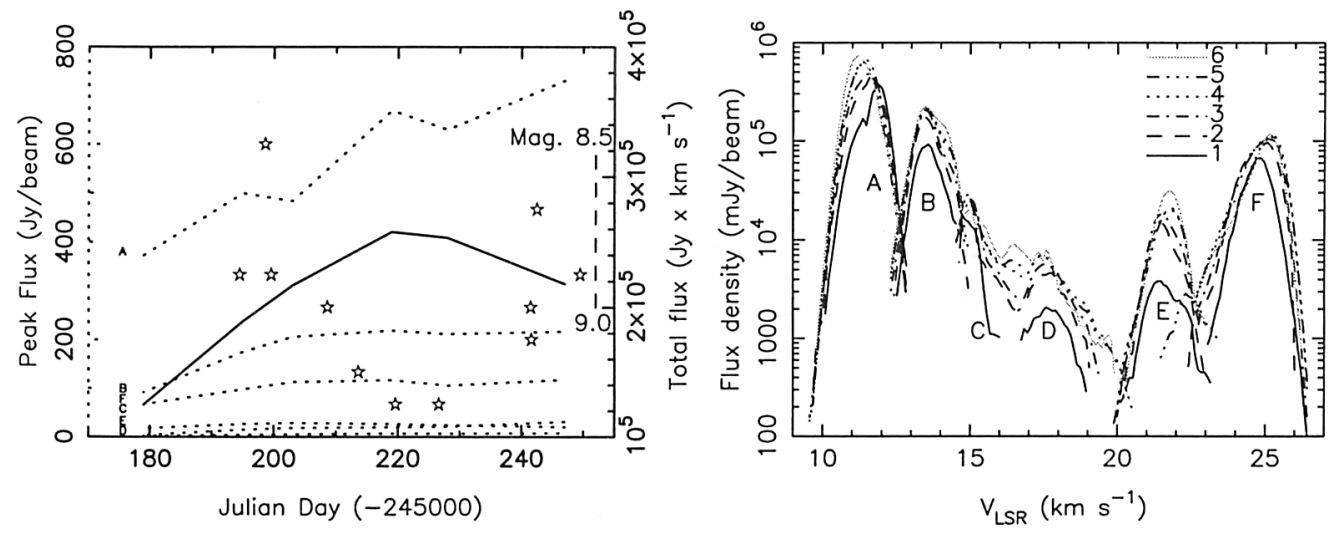

Figure 1. left (a) RT Vir total $22 \mathrm{GHz}$ flux density (solid line) and selected peaks of features A - F (dotted lines) at six epochs. The stars show the optical magnitude, scale is dashed line. right (b) Velocity profiles of features $\mathrm{A}-\mathrm{F}$ at epochs $1-6$.

resolution of $0.105 \mathrm{~km} \mathrm{~s}^{-1}$. 3C273 (flux density $31 \mathrm{Jy}$, Stevens et al. 1998) was used to establish the flux scale and correct the spectral bandpass at each epoch.

Table 1. MERLIN $22 \mathrm{GHz}$ observations of RT Vir since 1994

\begin{tabular}{|c|ccccccc|}
\hline Epoch & 1994 & 1 & 2 & 3 & 4 & 5 & 6 \\
JD 2400000+ & 49459 & 50179 & 50195 & 50203 & 50219 & 50228 & 50247 \\
Duration (hr) & 11.7 & 4.5 & 6.3 & 11.5 & 9.5 & 7.7 & 8.4 \\
\hline
\end{tabular}

At every epoch a map of the brightest channel of RT Vir was used to derive phase and amplitude corrections by self-calibration and the solutions were applied to all channels. The data cubes were mapped and CLEANed using a 12 mas diameter restoring beam. The off-source noise level was $\sigma_{\text {rms }} \approx 20$ $\mathrm{mJy} /$ beam. 2D Gaussian components were fitted to each patch of flux in each channel above the greater of $5 \sigma_{\text {rms }}$ or $2-4 \%$ of the peak. The relative position accuracy is proportional to the signal to noise ratio. Under these conditions it is typically 1 mas for a $1 \mathrm{Jy}$ component (Richards et al. 1998b; Condon 1997).

\section{Results}

\subsection{The RT Vir $\mathrm{H}_{2} \mathrm{O}$ maser shell}

Emission at $22 \mathrm{GHz}$ was detected between 9 and $27 \mathrm{~km} \mathrm{~s}^{-1}$. The brightest peak occurs between 11.27 and $11.52 \mathrm{~km} \mathrm{~s}^{-1}$ during these observations. It was used to align the epochs for this preliminary analysis. Fig. 1b shows the velocity profiles of selected features at all epochs and Fig. 2a shows emission integrated across all channels. Fig. $2 b$ shows the the flux density and velocity of all maser components at epoch (1). These Figures show that the brightest maser emission 

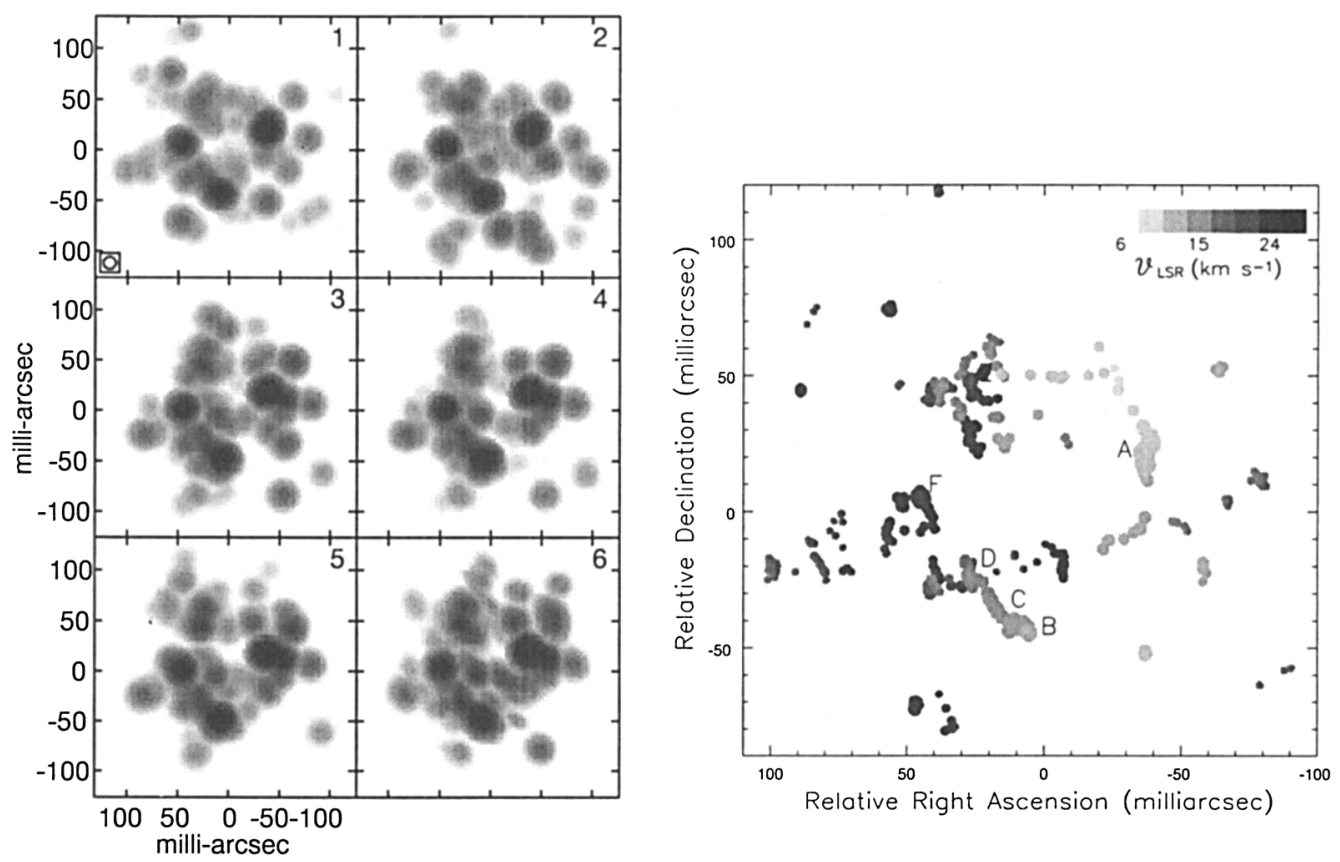

Figure 2. left (a) Maps of total $22 \mathrm{GHz}$ flux from RT Vir at epochs 1 6. right (b) Individual maser components at epoch 1. Symbol diameter is proportional to $\log$ (flux density) and the shade shows velocity.

forms a ring typical of tangential beaming due to an outflow accelerating radially away from the star (Chapman \& Cohen 1985). The centre of emission was found by assuming that the maser distribution forms a shell which is point-symmetric in space and velocity, this is at $(0,0)$ in Fig. 2.

Some red- and blue-shifted emission is seen in most parts of the shell, and emission close to $V_{*}$ is seen at large angular separations at all points of the compass. The simplest model is a thick spherical shell with inner and outer radii and expansion velocities marked in Fig. 3. These are respectively $r_{\mathrm{i}}=$ $6.9 \times 10^{11} \mathrm{~m} ; r_{\mathrm{o}}=28.8 \times 10^{11} \mathrm{~m} ; v_{\mathrm{i}}=3.5 \mathrm{~km} \mathrm{~s}^{-1} ; v_{\mathrm{o}}=11.5 \mathrm{~km} \mathrm{~s}^{-1}$. This gives a logarithmic velocity gradient $\epsilon \sim 0.8$.

However the outflow deviates from spherical symmetry. There is a SE NW offset between much of the red-shifted emission and much of the blueshifted emission. There is no evidence for any systematic rotation (Imai et al. 1997). Comparison of the maps and spectral profile of RT Vir with the models of Bowers (1991) shows that radial outflow, in which the velocity depends on direction alone, can produce an oblate ellipsoid (his figure $2, i=-45^{\circ}$, rotated by $45^{\circ}$ clockwise with respect to the observer). The appearance of RT Vir implies some modifications to this model. The most extreme red- and blue- shifted features have a large angular separation SE - NW but the whole shell has no obvious spatial asphericity despite a greater flux density in the SE and NW. It is possible that the star loses mass asymmetrically. A higher density in the 


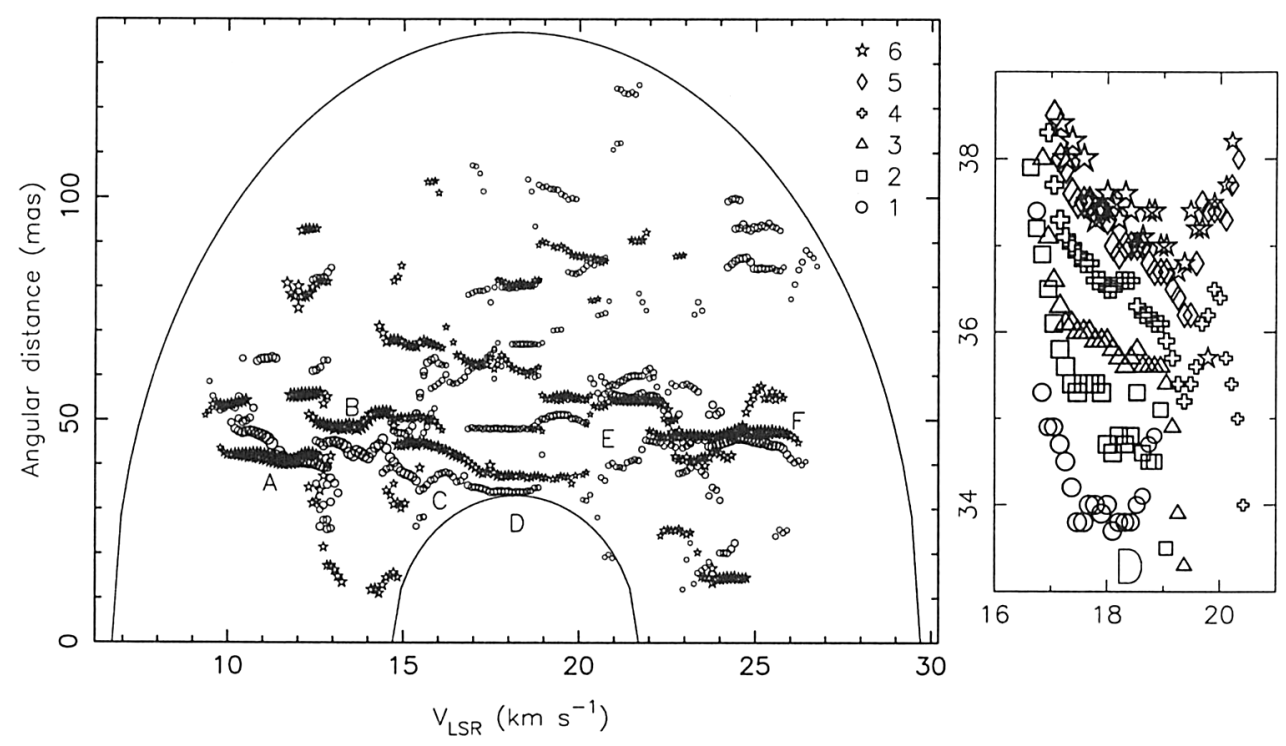

Figure 3. The angular separation of maser components from the assumed stellar position $a$ as a function of velocity; $V_{*}=18.2 \mathrm{~km} \mathrm{~s}^{-1}$. Epochs 1 and 6 are shown on the left; all epochs are shown for feature $\mathrm{D}$ on the right.

equatorial plane would be associated with more effective dust-gas momentum coupling, 'more acceleration and brighter emission in these directions.

\subsection{Maser clouds}

Fig. $2 \mathrm{~b}$ shows that in most cases the positions of components making up each feature shifts systematically with velocity. The average angular size of a feature is $11 \pm 5$ mas, corresponding to $2.3 \pm 1.0 \times 10^{11} \mathrm{~m}$. This represents the unbeamed size of maser clouds. Their radius is $\sim 6 \%$ of the mean radius of the RT Vir $\mathrm{H}_{2} \mathrm{O}$ maser shell. The measured radius of $\mathrm{H}_{2} \mathrm{O}$ clouds in the supergiant $\mathrm{S} \mathrm{Per}$ (Richards et al. 1998b) is the same fraction of its average shell radius although the scale is larger. $40-60$ features were identified in RT Vir at each epoch. The inner limit to $22 \mathrm{GHz}$ maser emission is determined by the maser quenching density, $5 \times 10^{15} \mathrm{~m}^{-3}$ at $1000 \mathrm{~K}$ (Cooke \& Elitzur 1985; Yates et al. 1997). However the number density at $r_{\mathrm{i}}$ derived from the $\mathrm{CO}$ mass loss rate (Bujarrabal et al. 1989 , adjusted to $140 \mathrm{pc}$ ) is only $\sim 10^{14} \mathrm{~m}^{-3}$, suggesting that the $\mathrm{H}_{2} \mathrm{O}$ masing clouds are 50 times denser than their surroundings, consistent with the findings for other late-type stars (Richards et al. 1996, 1998a, 1998b).

\subsection{Time variations}

The maps of the six epochs were made into a movie which shows small-scale shifts and brightening of the peaks, and the winking on and off of faint extended clouds. In Fig. 3 at least one new feature near $r_{\mathrm{i}}$ is only seen at later epochs; 
conversely some features near $r_{\mathrm{o}}$ are only seen at earlier epochs (Figs. 1a and 3), suggesting that new clouds enter the region suitable for masing as old ones leave.

Fig. 1a shows the variation with epoch of total $22 \mathrm{GHz}$ flux density. There is no obvious correlation with optical brightness. The flux density of the brightest features increases unsteadily throughout; the total flux peaks 20 days after the optical peak. The light travel time from RT Vir through the $\mathrm{H}_{2} \mathrm{O}$ maser shell is $\leq 3 \mathrm{hr}$. The dust is directly heated by absorbing stellar radiation, which also provides radial acceleration. The gas is in turn heated and accelerated by collisions. If this involved a delay of several weeks then the denser regions nearest the star would brighten first. There is no clear evidence for this, and it is likely that the propagation of shocks and changes in the velocity gradient are important.

Some preliminary quantitative results were obtained by comparing six features which are clearly defined at each epoch. These are labelled $\mathrm{A}-\mathrm{F}$ in the figures. Table 2 lists the flux-weighted mean Doppler velocity of each feature $\left(u=V_{\mathrm{LSR}}-V_{*}\right)$; the error-weighted mean angular separation from the assumed stellar position $(a)$ and the peak flux $(P)$; the errors are less than a few percent. An overline denotes the mean value for six epochs and prefix $\delta$ is the greatest change. An asterisk shows that this is not between the first and last epochs. $\delta P \%$ is $\delta P / P($ epoch 1$)$. The mean angular sizes and velocity width of the features $(\bar{L}$ and $\overline{\Delta V})$, do not show any systematic variation with time.

Table 2. Six $\mathrm{H}_{2} \mathrm{O}$ maser features in RT Vir (see text for definitions).

\begin{tabular}{|c|c|c|c|c|c|c|c|c|c|}
\hline Feature & $\left(\mathrm{km} \mathrm{s}^{-1}\right)$ & $\left.\mathrm{s}^{-1}\right)^{\delta u}$ & \multicolumn{2}{|c|}{ (mas) } & $\bar{P}$ & у) $\delta P$ & $\begin{array}{r}\delta P \% \\
\%\end{array}$ & $\begin{array}{c}\bar{L} \\
\text { (mas) }\end{array}$ & $\begin{array}{c}\overline{\Delta v} \\
\left(\mathrm{~km} \mathrm{~s}^{-1}\right)\end{array}$ \\
\hline $\mathrm{A}$ & -6.8 & -0.43 & 42 & -1 & 550 & +357 & $96 \%$ & $18 \pm 5$ & $3.1 \pm 0.2$ \\
\hline B & -4.6 & +0.07 & 48 & +6 & 150 & +125 & $136 \%$ & $11 \pm 3$ & $2.4 \pm 0.2$ \\
\hline $\mathrm{C}$ & -2.9 & +0.68 & 40 & +6 & 24 & $* 11$ & $* 62 \%$ & $15 \pm 3$ & $2.0 \pm 0.3$ \\
\hline D & -0.4 & $*+0.27$ & 36 & +3 & 5 & +6 & $288 \%$ & $12 \pm 2$ & $2.9 \pm 0.5$ \\
\hline $\mathrm{E}$ & +3.5 & $*+0.16$ & 52 & $* \pm 3$ & 20 & +27 & $715 \%$ & $13 \pm 7$ & $3.0 \pm 0.3$ \\
\hline $\mathrm{F}$ & +6.6 & +0.17 & 45 & $*+3$ & 93 & +51 & $76 \%$ & $16 \pm 5$ & $3.9 \pm 0.6$ \\
\hline
\end{tabular}

The mean increase in separation between all pairs of the six features is not affected by alignment uncertainties and is $3.0 \pm 0.5$ mas in 68 days, corresponding to $11 \pm 2 \mathrm{~km} \mathrm{~s}^{-1}$ if RT Vir is at $140 \mathrm{pc}$. The features lie $36-52$ mas from RT Vir, so for uniform acceleration the expansion velocity in this region should be $\leq 5 \mathrm{~km} \mathrm{~s}^{-1}$. Fig. $1 \mathrm{~b}$ shows that for features with larger values of $|u|$ the lines as a whole move towards higher outflow velocities even though $\delta u$ is small. These bright features may be denser, dustier and more effectively accelerated than their surroundings. Alternatively the apparent outward proper motion of the brightest emission may be a pattern motion of optimum masing conditions (e.g. the passage of shocks).

RT Vir was observed by MERLIN under similar conditions in 1994 (Bains 1995) and showed a similar structure in the CSE as a whole, with brighter blue- 
shifted emission and the SE - NW velocity asymmetry; however the brightest features were in different locations and if any features had survived their internal structure and brightness were very different. There are even greater differences with earlier maps by Yates \& Cohen (1994) and Bowers et al. (1993).

\section{Conclusions}

The $22 \mathrm{GHz} \mathrm{H}_{2} \mathrm{O}$ emission from around RT Vir forms an expanding shell with a velocity gradient $\epsilon \approx 0.8$. Emission is brightest in the $\mathrm{SE}$ (red-shifted) and NW (blue-shifted) suggesting an elliptical velocity field. Individual masing clouds are typically $\sim 2.3 \times 10^{11} \mathrm{~m}$ in diameter and span $1-3 \mathrm{~km} \mathrm{~s}^{-1}$. Many individual features are recognisable for at least 10 weeks but their flux changes severalfold. There is no obvious correlation with stellar optical magnitude. The brightest show proper motions 1 or 2 times the uniform acceleration model shell expansion velocity. Further analysis is underway to establish the physical conditions in the masing regions and to investigate the rôle of the stellar brightness, pulsations, uneven mass loss, and so on, in determining the appearance of the masers.

\section{References}

Bains I., 1995, Master's Thesis, University of Manchester

Bowers P.F., 1991, ApJS 76, 1099

Bowers P.F., Claussen M.J., Johnston K.L., 1993, AJ 105, 284

Bujarrabal V., Gómez-González J., Planesas P., 1989, A\&A 219, 256

Chapman J.M., Cohen R.J., 1985, MNRAS 212, 375

Condon J.J., 1997, PASP 109, 166

Cooke B., Elitzur M., 1985, ApJ 295, 175

Imai H., Shibata K.M., Sasao T., et al., 1997, A\&A 319, L1

Kholopov P.N., et al., 1985, GCVS, Nauka

Kirrane M.-T., 1986, Master's Thesis, University of Manchester

Mendoza-Torres J.E., Lekht E.E., Berulis I.I., Pashchenko M.I., 1997, A\&AS 126,257

Nyman L.-Å., Johansson L.E.B., Booth R.S., 1986, A\&A 160, 352

Richards A.M.S., Yates J.A., Cohen R.J., 1996, MNRAS 282, 665

Richards A.M.S., Yates J.A., Cohen R.J., 1998a, MNRAS 299, 319

Richards A.M.S., Yates J.A., Cohen R.J., 1998b, MNRAS, submitted

Stanek K.Z., Knapp G.R., Young K., Phillips T.G., 1995, ApJS 100, 169

Stevens J.A., Robson E.I., Gear W.K., et al., 1998, ApJ 502, 182

Szymczak M., Le Squeren A.M., 1998, A\&A, submitted

Whitelock P.A., Menzies J., Feast M., et al., 1994, MNRAS 267, 711

Yates J.A., Cohen R.J., 1994, MNRAS 270, 958

Yates J.A., Field D., Gray M.D., 1997, MNRAS 285, 383 